\title{
A retroperitoneal supra-adrenal non- communicating gastric duplication cyst: a case report and comprehensive literature review
}

Mahmood Masud Al Awfi ${ }^{{ }^{*}}$ (D), Zainab Nasser Al Balushi ${ }^{2}$, Suad Rashid Al Jahdhami ${ }^{2}$, Mahmoud Hatem Sherif ${ }^{2}$ and Sareyah Al Sibai

\begin{abstract}
Background: Gastric duplication cyst is a rare congenital anomaly. It is usually located in close proximity to the stomach. Nevertheless, there have been seldom reports in the literature that describe unusual locations. Retroperitoneal gastric duplications cysts are extremely rare and are usually confused with renal or adrenal cysts.

Case presentation: In this case report, we are presenting a 2-year-old girl with a complaint of bouts of abdominal pain, found to have a retroperitoneal supra-adrenal cyst on imaging. She underwent laparoscopic resection of the cyst with uneventful post-operative course. The histopathological diagnosis was surprisingly gastric duplication cyst.

Conclusion: In our review of the literature, the variability of this condition and its clinical manifestation are apparent. We also conclude that this diagnostic entity should be part of the differential diagnosis of intraperitoneal or retroperitoneal cysts and that laparoscopic resection of retroperitoneal cysts is the preferred approach.
\end{abstract}

Keywords: Gastric duplication cyst, Enteric duplication, Retroperitoneal cyst, Congenital cyst

\section{Background}

Gastric duplication cyst is a rare congenital anomaly. These cysts are usually lined with gastric mucosa and have all the layers of the stomach. In addition, it may or may not communicate with the organ of origin. Moreover, a gastric duplication cyst is usually located in close proximity to the stomach. Retroperitoneal gastric duplication cyst mimicking renal or adrenal cysts are rarely reported in the literature [1-3]. Therefore, we are reporting our case in addition to literature review on enteric duplications with focus on retroperitoneal gastric duplication.

\footnotetext{
*Correspondence: mahmood.alawfi@gmail.com

'Department of Surgery, Oman Medical Speciality Board, Muscat, Sultanate of Oman

Full list of author information is available at the end of the article
}

\section{Case presentation}

A 2-year-old girl was referred to our service for evaluation of a renal cyst. The cyst was first noted in the antenatal scan, which was confirmed later in a post-natal scan. The cyst initially measured $4.4 \mathrm{~cm}$. At the age of two, she presented with bouts of abdominal pain. Her clinical examination was unremarkable and her laboratory workup was normal apart from mild anemia. An abdominal CT-scan was done which showed a left supra-renal cyst, measuring $5.4 \times 5.4 \times 4.8 \mathrm{~cm}$. It was in the superior pole of the left kidney, abutting the pancreas, and the medial aspect of the spleen (Fig. 1). The impression was of a benign adrenal cyst, and she was booked for laparoscopic adrenalectomy.

The intra-operative findings were of a left adrenal cyst attached to the tail of the pancreas, splenic vessels, and the posterior wall of the abdomen. The cyst was resected and sent for histopathological evaluation (Fig. 2). The intra- 


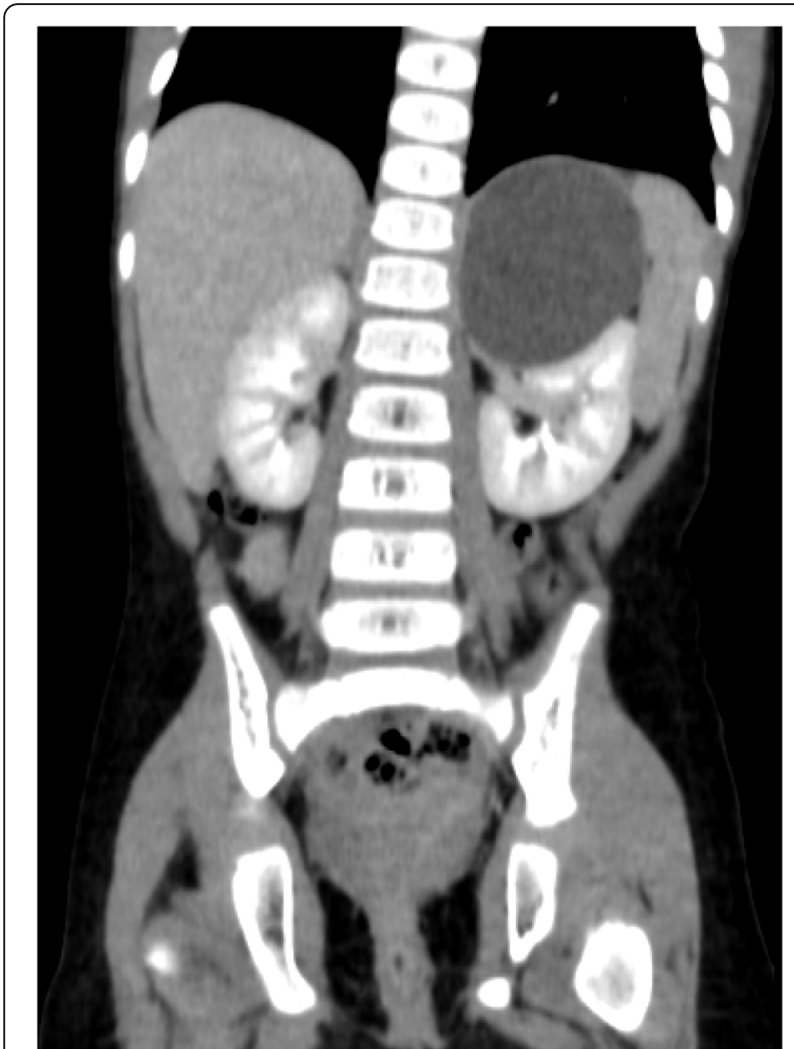

Fig. 1 A contrast enhanced image from a computerized tomography $(\mathrm{CT})$ scan of the chest and abdomen

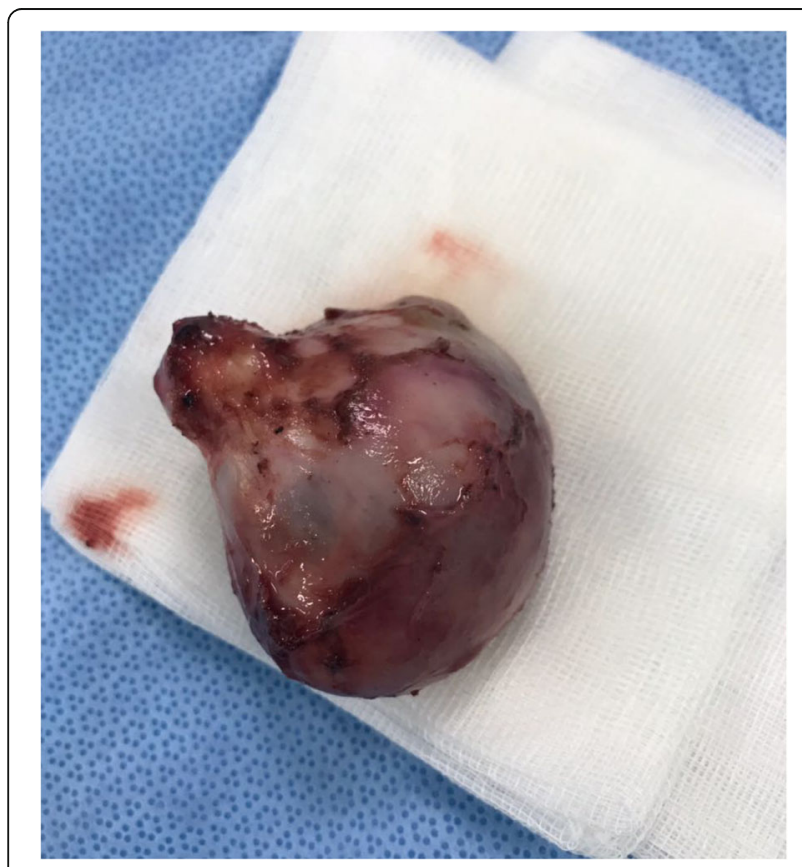

Fig. 2 Gastric duplication cyst specimen operative and post-operative periods were uneventful, and the patient was discharged on the first day post-operation.

The specimen revealed a cyst wall composed of all four layers of the stomach including specialized gastric mucosa, sub-mucosa, muscularis propria, and adventitia. There was patchy chronic inflammation, fibrosis, erosion, and thinning of the wall. No adrenal or renal tissues were identified and no other endodermal or ectodermal derivatives were seen. There was no evidence of malignancy (Fig. 3). The final histopathological diagnosis was consistent with a gastric duplication cyst. On follow-up, she was doing well and pain-free.

\section{Discussion}

Enteric duplication (ED) cyst are rare congenital anomalies. This diagnostic entity was first described in 1733 by Calder [4]. The incidence of this condition is not accurately known, but it has been reported to be 1 in 4500 autopsy series [4]. W. E. Ladd published in 1937 an article to universally apply the term Duplications of the Alimentary Tract, i.e., enteric duplications, for any congenital lesion if it is lined with gastrointestinal tract (GIT) epithelium, covered with a smooth muscle layer and its anatomical location is near an organ of the GIT $[4,5]$. However, the current literature data did provide some exceptions with a logical explanation found in relation to embryogenesis and organogenesis. For example, gastric duplications cyst might be lined with respiratory epithelium, in view of the fact that gastric epithelium and lower respiratory epithelium are the differentiation of the foregut endoderm [6].

The basis of ED development is not yet factualized, but there are few explanative theories. The split notochord concept is by far the most acknowledged. Incomplete twinning, phylogenetic reversal, persistent embryonic diverticula, entrapment of endodermal cells or persistent epithelial buds within the body wall and dysvacuolation are other theories that have been suggested [7].

Duplications can be cystic, tubular, or mixed. The tubular form is rare and forms only $10 \%$ of all ED [8]. It can have foregut, midgut, or hindgut origin. The location according to the literature may be from the oral cavity to the anus $[4,5]$. The ileal origin is the most common. Five of the largest case series of enteric duplication cysts were reviewed and the organ of origin in terms of frequency was as follows: ileal, colonic, gastric, duodenal, esophageal followed by others [5, 9-12].

Most case series show that males are predominantly affected [4, 9, 11]. Patients may be asymptomatic and if they exhibit symptoms it is usually before the age of 2 years [4]. They have variable clinical presentations depending on the type of duplication, shape, location, communication to GIT, and presence of ectopic tissues. The variability is wide clinically and radiologically, which 


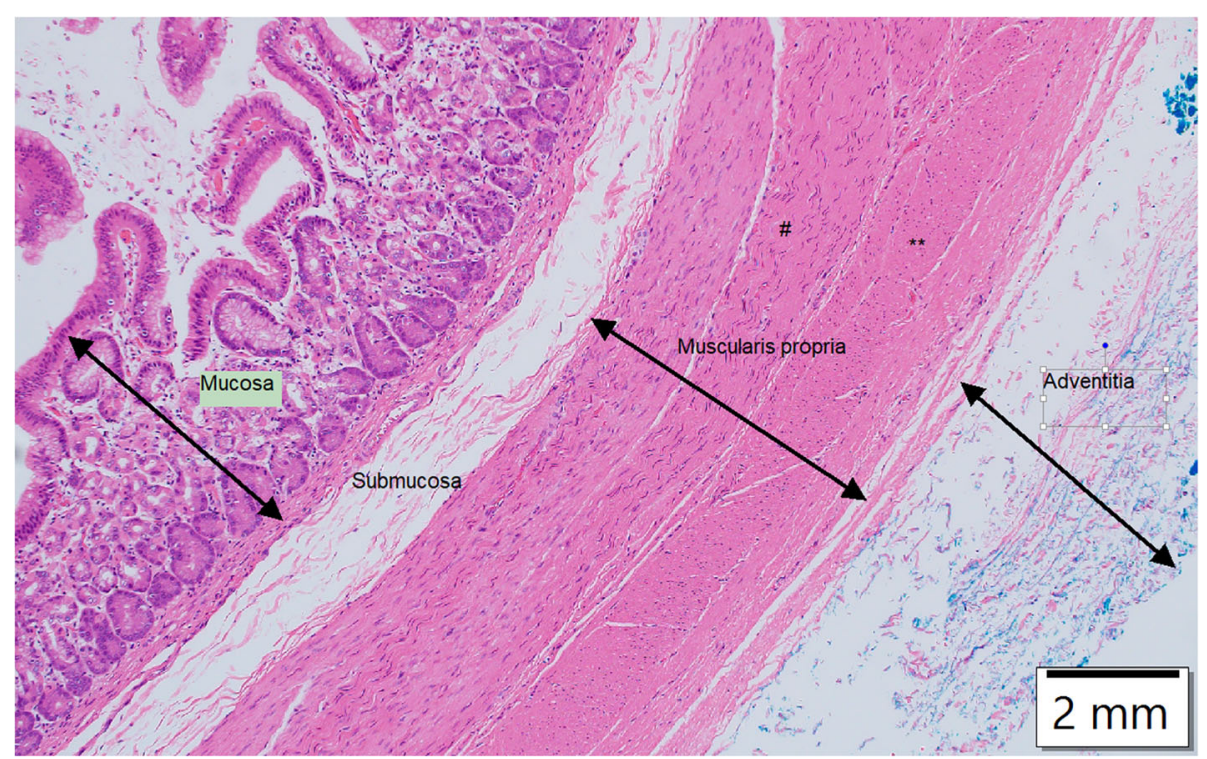

Fig. 3 Hematoxylin and eosin (H\&E) stained section of cyst wall showing all four stomach layers including; lining of specialized gastric mucosa, submucosa, muscularis propria (both inner circular" and outer longitudinal**) and adventitia [× 100]

can be confused with other GIT pathology; thus, a high index of suspicion should be present. They may present with abdominal pain, abdominal mass, obstruction, perforation, rectal bleeding, melena, or the clinical presentation may be specific to the location. For example, thoracic duplication cyst may present with pneumonia, respiratory obstruction, aspiration, or cough. With duodenal duplication cyst, jaundice could be a presenting symptom. Further, bowel duplications cyst may present with intussusception or volvulus $[5,10]$.

In enteric duplication patient, the associated congenital anomalies reported to reach up to 50\% [4]. Moreover, skeletal anomalies frequently found in foregut duplication patient whereas gastrointestinal or genitourinary tracts malformation were noted more commonly in patient with midgut and hindgut duplication. Nevertheless, vertebra anomalies are the most common anomalies found overall $[4,13]$.

Duplications are diagnosed based on imaging findings, starting with ultrasonography then followed by CT-scan for more detailed anatomical correlation. If there is suspicion of tubular lesion then CT-scan is preferred on those cases. Other imaging studies can be used on case to case basis, such as MRI, fluoroscopy, or others [14]. Pre-natal diagnosis has been reported in the literature using ultrasonography, but post-natal diagnosis is the usual norm [15]. Cystic lesion is described as anechoic cyst, with gut signature sign denotating a multilayeredwall with echogenic internal mucosa layer and hypoechoic external muscular layer. Though, the realtime image provided by the sonographic scan can elicit peristalsis of the cyst unlike other imaging modalities; however, its diagnostic property is operator dependent [16].

Endoscopic procedure utilization has been reported in the literature for diagnostic and therapeutic purposes. However, the current evidence is in favor of utilizing it for non-intervention-based diagnoses such as esophagogastroduodenoscopy, colonoscopy, or endoscopic ultrasonography. Interventional procedures such as fine-needle aspiration of the cyst endoscopically are not advisable because of the infective complication. As for its therapeutic use, there is no strong evidence to supports its routine use in terms of efficacy and safety nor its outcomebasedsuperiority to surgery. Though, this line of management can be considered once surgery is not an option [17, $18]$.

In asymptomatic patients with incidental diagnosis, the management options are either to observe or to surgically resect the lesion. However, there is no consensus and both options have been advocated in the literature.

In symptomatic patients, complete surgical excision is the treatment of choice. The basic goal is to alleviate the symptoms while providing the best outcome to the patient. Therefore, partial excision with mucosectomy and internal drainage have been advocated also when complete excision is not possible, to obtain a desirable outcome without compromising the patients' quality of life $[18,19]$.

Our duplication case was diagnosed histologically to be of gastric origin, and these are known to be cystic in nature. They have female predominance (8:1), and they are most commonly diagnosed within the first 3 months of life. The distal greater curvature duplication has the 
highest occurrence, and they are more commonly noncommunicating type [20]. Gastric duplication cyst (GDC) can be intra-peritoneal or retroperitoneal [1]. GDC can present with different clinical manifestations, often with abdominal mass, gastrointestinal obstruction, and anemia, especially in the initial first year of life. Gastrointestinal bleeding is a less frequent presentation and hematemesis is a rare presentation. As the child grows, abdominal pain becomes more common, and it can be due to many causes including ulceration of the cyst, rupture, infection, over distention of the cyst, or due to secondary pancreatitis [21, 22].

Retroperitoneal duplication is rare and only few cases have been reported. The majority have been diagnosed prenatally or postnatally with ultrasonography. Many were asymptomatic. If not diagnosed early, then chronic dull abdominal pain or obstructive compression maybe their clinical manifestation. Another unusual presentation has been described if the cyst fistulizes to another organ [1, 2, 23]. Radiographic imaging has a key role in diagnosing GDC, namely ultrasonography and CT-scan. Moreover, technetium T99 scan can be used to detect ectopic gastric mucosa in those cysts to aid the diagnosis [24].

Surgical resection can be performed via open or laparoscopic approach. Recently, robotic-assisted resection of GDC has been reported [25]. Trans-peritoneal and extra-peritoneal approach can be used for resection of retroperitoneal GDC. Surgeon experience, hospital setting, cyst anatomic co-relation, patient status, and preference all play a role in choosing the approach. However, minimal access surgery should be considered first because of its superior clinical outcome with reasonable theoretical explanation. In addition, laparoscopy has a diagnostic benefit that can be of help in doubtful cases $[2,3]$.

\section{Conclusion}

In conclusion, enteric duplication is a challenging diagnostic entity with numerous presentations and manifestation requiring different diagnostic approaches personalized case to case. Surgery is the mainstay of treatment and laparoscopy is the approach of choice. However, preoperative planning is necessary to avoid unexpectable obstacles and to ensure better outcome. Retroperitoneal gastric duplication is no different.

\footnotetext{
Abbreviations

ED: Enteric duplication; GIT: Gastrointestinal tract; GDC: Gastric duplication cyst
}

\section{Acknowledgements}

Not applicable

Randomization

Not applicable

Double blinded

Not applicable

\section{Authors' contributions}

All authors approved submission and agreed to be accountable for the publication, with the following contribution: MMA: conception + design of the work + acquisition + have drafted the work + substantively revised it: ZNB: conception + design of the work + substantively revised it: SRJ: have drafted the work (pathology related) + substantively revised it: MHS: design of the work + substantively revised it: SS: conception + design of the work + acquisition.

Funding

Not applicable

Availability of data and materials

Available. Available upon request

Ethics approval and consent to participate

Approved

Consent for publication

Taken

Competing interests

The authors declare no competing interests.

\section{Author details}

'Department of Surgery, Oman Medical Speciality Board, Muscat, Sultanate of Oman. ${ }^{2}$ Sultan Qaboos University Hospital, Al Seeb, Oman.

Received: 5 August 2020 Accepted: 8 February 2021

Published online: 12 April 2021

\section{References}

1. Chen PH, Lee JY, Yang SF, Wang JY, Lin JY, Chang YT. A retroperitoneal gastric duplication cyst mimicking a simple exophytic renal cyst in an adolescent. J Pediatr Surg. 2010;45(10):e5-8.

2. Pachl M, Patel K, Bowen C, Parikh D. Retroperitoneal gastric duplication cyst: a case report and literature review. Pediatr Surg Int. 2012;28(1):103-5.

3. Castillo-Fernández AL, Vázquez-Rueda F, Cañete MD, Caballero-Villarraso J. Retroperitoneal gastric duplication mimicking a prenatal adrenal cyst. Congenit Anom (Kyoto). 2018;58(4):141-2. https://doi.org/10.1111/cga.12244.

4. Liaqat N, Latif T, Khan FA, Iqbal A, Nayyar SI, Dar SH. Enteric duplication in children: a case series. Afr J Paediatr Surg. 2014;11:211-4.

5. Bower RJ, Sieber WK, Kiesewetter WB. Alimentary tract duplications in children. Ann Surg. 1978;188(5):669-74.

6. Tjendra Y, Lyapichev K, Henderson J, Rojas CP. Foregut duplication cyst of the stomach: a case report and review of the literature. Case Rep Pathol. 2016;2016:7318256.

7. Tiwari C, Shah H, Waghmare M, Makhija D, Khedkar K. Cysts of gastrointestinal origin in children: varied presentation. Pediatr Gastroenterol Hepatol Nutr. 2017;20(2):94-9.

8. Hitch DC, Shandling B, Gilday DL. Tubular duplication of the bowel. Use of technetium $99 \mathrm{~m}$ pertechnetate in diagnosis. Arch Dis Child. 1978; 53(2):178-9.

9. Erginel B, Soysal FG, Ozbey H, Keskin E, Celik A, Karadag A, et al. Enteric duplication cysts in children: a single-institution series with forty patients in twenty-six years. World J Surg. 2017:41(2):620-4.

10. Okur MH, Arslan MS, Arslan S, Aydogdu B, Türkçü G, Goya C, et al. Gastrointestinal tract duplications in children. Eur Rev Med Pharmacol Sci. 2014;18(10):1507-12.

11. Lima M, Molinaro F, Ruggeri G, Gargano T, Randi B. Role of mini-invasive surgery in the treatment of enteric duplications in paediatric age: a survey of 15 years. Pediatr Med Chir. 2012;34(5):217-22.

12. Laje P, Flake AW, Adzick NS. Prenatal diagnosis and postnatal resection of intraabdominal enteric duplications. J Pediatr Surg. 2010;45(7):1554-8.

13. Ildstad ST, Tollerud DJ, Weiss RG, Ryan DP, McGowan MA, Martin LW. Duplications of the alimentary tract. Clinical characteristics, preferred treatment, and associated malformations. Ann Surg. 1988;208(2):184-9.

14. Laskowska K, Gałązka P, Daniluk-Matraś I, Leszczyński W, Serafin Z. Use of diagnostic imaging in the evaluation of gastrointestinal tract duplications. Pol J Radiol. 2014;79:243-50. 
15. Nakazawa N, Okazaki T, Miyano T. Prenatal detection of isolated gastric duplication cyst. Pediatr Surg Int. 2005;21(10):831-4.

16. Di Serafino M, Mercogliano C, Vallone G. Ultrasound evaluation of the enteric duplication cyst: the gut signature. J Ultrasound. 2015;19(2):131-3.

17. Romeo E, Torroni F, Foschia F, De Angelis P, Caldaro T, Santi MR, et al. Surgery or endoscopy to treat duodenal duplications in children. J Pediatr Surg. 2011;46(5):874-8.

18. Liu R, Adler DG. Duplication cysts: diagnosis, management, and the role of endoscopic ultrasound. Endosc Ultrasound. 2014;3(3):152-60.

19. Jehangir S, Ninan PJ, Jacob TJ, Eapen A, Mathai J, Thomas RJ, et al. Enteric duplication in children: experience from a tertiary center in South India. J Indian Assoc Pediatr Surg. 2015;20(4):174-8.

20. Doepker MP, Ahmad SA. Gastric duplication cyst: a rare entity. J Surg Case Rep. 2016;2016(5):rjw073. https://doi.org/10.1093/jscr/rjw073.

21. Bailey CE, Fritz MB, Webb L, Merchant NB, Parikh AA. Gastric duplication cyst masquerading as a mucinous pancreatic cyst: case report and literature review. Ann R Coll Surg Engl. 2014;96(1):88E-90E.

22. Surridge CA, Goodier MD. Gastric duplication cyst: a cause of rectal bleeding in a young child. Afr J Paediatr Surg. 2014;11(3):267-8.

23. Bal HS, Kisku S, Sen S, Masih D. A retroperitoneal enteric duplication cyst communicating with the right upper ureter in an infant. BMJ Case Rep. 2014;2014:bcr2013202449. https://doi.org/10.1136/bcr-2013-202449.

24. Zhang L, Chen Q, Gao Z, Xiong Q, Shu Q. Diagnosis and treatment of gastric duplication in children: a case report. Exp Ther Med. 2017;14(4): 3062-6.

25. Rizzo R, Lisi G, Marino N, Lauriti G, Di Renzo D, Lelli Chiesa P. Robot-assisted resection of gastric duplication cysts in a child: a detailed case report. La Pediatria Medica E Chirurgica. 2018;40(2):52-5. https://doi.org/10.4081/pmc.2 018.203 .

\section{Publisher's Note}

Springer Nature remains neutral with regard to jurisdictional claims in published maps and institutional affiliations.

\section{Submit your manuscript to a SpringerOpen ${ }^{\circ}$ journal and benefit from:}

- Convenient online submission

- Rigorous peer review

- Open access: articles freely available online

- High visibility within the field

- Retaining the copyright to your article

Submit your next manuscript at $\boldsymbol{\nabla}$ springeropen.com 